\title{
Technological developments toward the Small Size Telescopes of the Cherenkov Telescope Array
}

\author{
R. Canestrari ${ }^{* a}$, T. Greenshaw ${ }^{b}$, G. Pareschi ${ }^{a}$ and R. White ${ }^{c}$ for the CTA Consortium \\ ${ }^{a}$ INAF-Osservatorio Astronomico di Brera - Via Bianchi, 4623807 Merate (Lc) Italy \\ ${ }^{\mathrm{b}}$ University of Liverpool, Liverpool, UK \\ ${ }^{\mathrm{c}}$ University of Leicester, Leicester, UK
}

\begin{abstract}
In the last two decades a new window for ground-based high energy astrophysics has been opened. This explores the energy band from about $100 \mathrm{GeV}$ to $10 \mathrm{TeV}$ by making use of Imaging Atmospheric Cherenkov Telescopes (IACTs). Research in Very High Energy (VHE) gamma-ray astronomy is progressing rapidly and, thanks to the newest facilities such as MAGIC, HESS and VERITAS, astronomers and particle physicists are obtaining data with far-reaching implications for theoretical models.

The Cherenkov Telescope Array (CTA) is the ambitious international next-generation facility for gamma-ray astronomy and astrophysics that aims to provide a sensitivity of a factor of 10 higher than current instruments, extend the energy band coverage from below $50 \mathrm{GeV}$ to above $100 \mathrm{TeV}$, and improve significantly the energy and angular resolution to allow precise imaging, photometry and spectroscopy of sources. To achieve this, an extended array composed of nearly 100 telescopes of large, medium and small dimensions is under development. Those telescopes will be optimized to cover the low, intermediate and high energy regimes, respectively.

In this paper, we focus our attention on the Small Size Telescopes (SSTs): these will be installed on the CTA southern hemisphere site and will cover an area of up to $10 \mathrm{~km}^{2}$. The energy range over which the SSTs will be sensitive is from around $1 \mathrm{TeV}$ to several hundreds of $\mathrm{TeV}$. The status of the optical and mechanical designs of these telescopes is presented and discussed. Comments are also made on the focal surface instruments under development for the SSTs.
\end{abstract}

Keywords: CTA, Imaging Atmospheric Cherenkov Telescope, gamma-rays, segmented optics

\section{INTRODUCTION}

With the advent of ground-based IACTs in late 1980's, observations of VHE gamma-rays became possible and, since the discovery of the TeV emission from the Crab Nebula by Whipple in 1989 [1], this astronomy has achieved exceptional results. More then $130 \mathrm{TeV}$ sources, both galactic and extragalactic, have been detected to date (see Figure 1).

The international communities working in TeV astronomy in Europe, America and Japan are now involved in the study of a huge array of Cherenkov telescopes called the Cherenkov Telescope Array [2] observatory. CTA aims to (a) increase sensitivity by another order of magnitude for deep observations, (b) boost significantly the detection area and hence the detection rates, particularly important for transient phenomena and at the highest energies, (c) improve the angular resolution and hence the ability to resolve the morphology of extended sources, (d) provide wide and uniform energy coverage from some $10 \mathrm{GeV}$ to beyond $100 \mathrm{TeV}$ in the energy of the photons, and (e) enhance the all sky survey capability, the monitoring capability and the flexibility of operation.

${ }^{*}$ rodolfo.canestrari@brera.inaf.it

Ground-based and Airborne Telescopes IV, edited by Larry M. Stepp, Roberto Gilmozzi, Helen J. Hall, Proc. of SPIE Vol. 8444, 844439 - (c) 2012 SPIE · CCC code: 0277-786/12/\$18 - doi: 10.1117/12.925562 

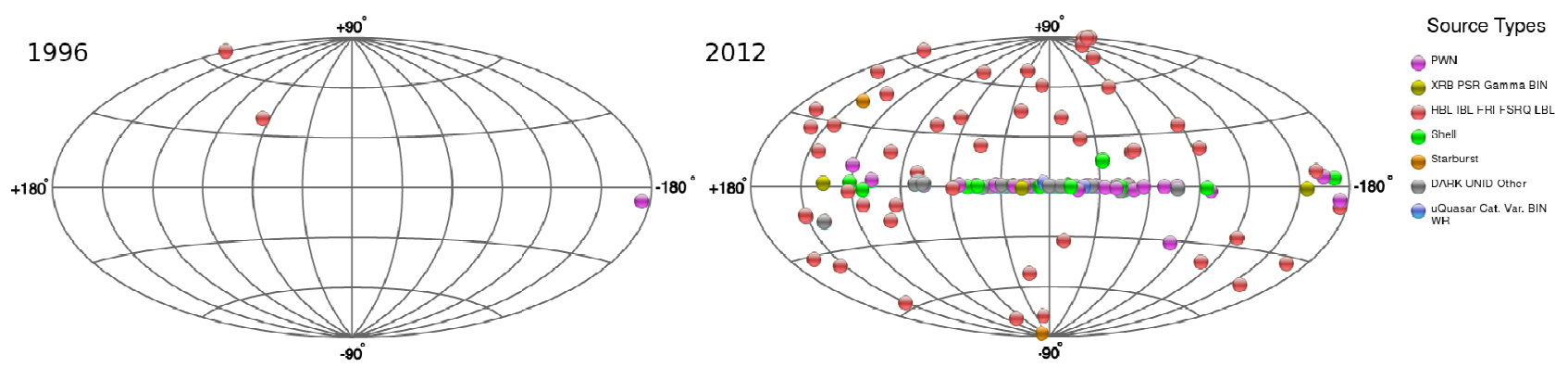

Figure 1. Sky maps showing the improvement in number of sources detected in the VHE astrophysics from 1996 (left panel) to 2012 (right panel). (Images created using http://tevcat.uchicago.edu/)

CTA will implement a large array with a modular design composed of three different telescope types, namely the Small Size Telescopes (SST), the Medium Size Telescopes (MST) and Large Size Telescopes (LST), of 4-7m, 12m, and 24m diameter respectively. An artist's view of CTA is represented in Figure 2.

The SSTs are the component of CTA that will allow observation and measurement of photons in the energy range from about $1 \mathrm{TeV}$ to $300 \mathrm{TeV}$. The lower limit of this range is determined by the threshold at which the SSTs trigger and is matched to the sensitivity of the MST section of CTA, while the upper limit is given by the area of the array on the ground, which must be of order $10 \mathrm{~km}^{2}$ to achieve the high energy differential sensitivity required by CTA. The performance of this section of the array in terms of angular and energy resolution is $0.04^{\circ}$ and $10 \%$, respectively. These are achievable by the deployment of an array populated by many tens of telescopes (40-60 units) exploiting large fields of view of the order of $8^{\circ}-10^{\circ}$ allowing the imaging of showers whose axis is far from a given telescope.

CTA was listed in the emerging proposals of ESFRI 2006 and it is now ranked in ASPERA and ASTRONET. Moreover, in December 2009, the European Commission within the 7th Framework Program (FP7) provided funding to the CTA Consortium, comprising 129 institutes from 22 countries, for the Preparatory Phase of the Cherenkov Telescope Array (CTA-PP, FP7-INFRASTRUCTURES-2010-1).

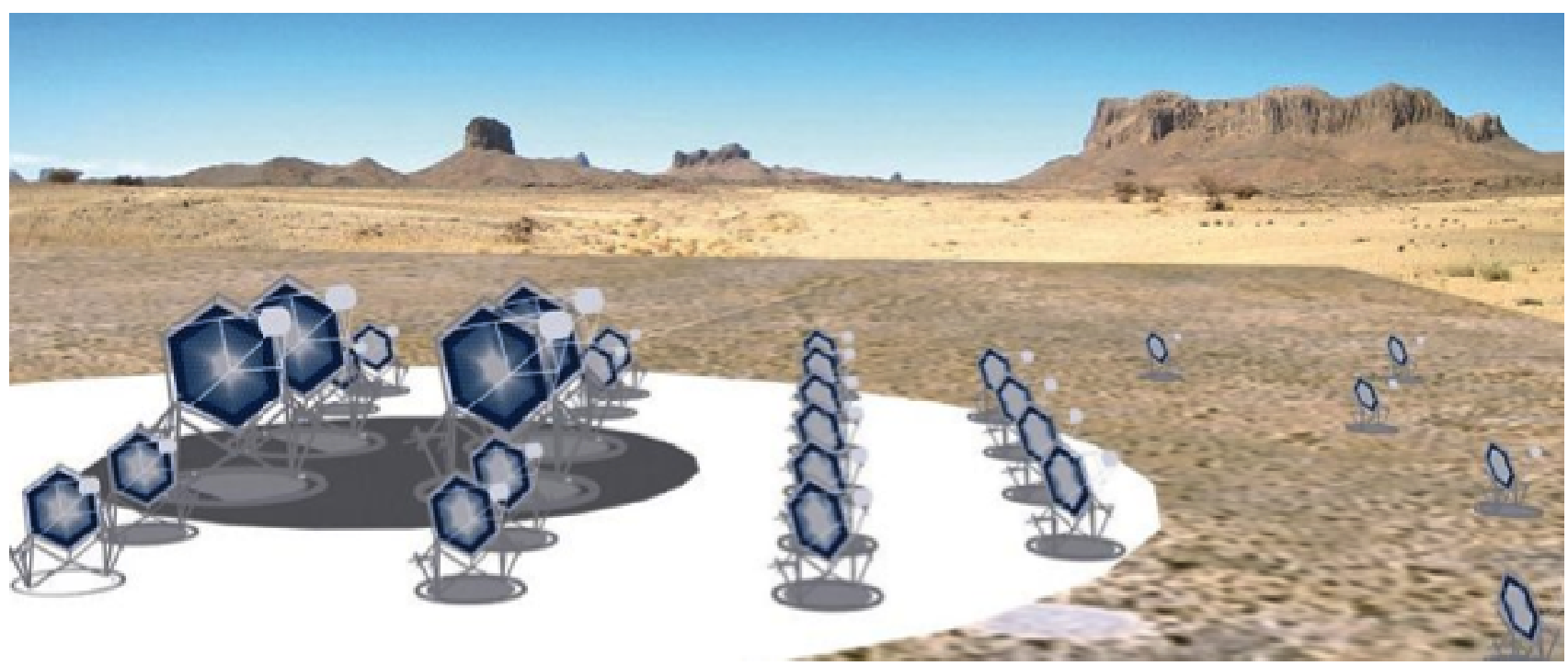

Figure 2. Artist's view of a possible CTA configuration, with three different telescopes types covering overlapping energy ranges, and area coverage which increases with increasing gamma-ray energy. 
This new, complex and very large facility needs, amongst other things, a substantial technological development of many telescope subsystems. From the installation, operation and maintenance of each single telescope to the efficient coordination of the complete array in a structure organized as an observatory, from the science data center to the huge power supply.

Within the framework presented above, this paper has a particular focus on the development of the SST section of the CTA observatory. We describe the design of the telescope optics and structure and discuss the mirror and the camera systems for the different telescope options considered.

\section{SINGLE-REFLECTION TELESCOPES: THE DAVIES-COTTON DESIGN}

The SST array can be constructed in several alternative ways. The first approach, which is that traditionally used for IACTs, uses Davies-Cotton optics (SST-DC). The attraction of this approach is that it is a proven technology and allows the use of a camera based on conventional photomultipliers (PMTs) which is very similar to that needed for the MST. It is, however, possible that this is not the best way for CTA to proceed. As the camera dominates the cost of telescopes of this size, large numbers of SSTs-DC could not be easily affordable.

\subsection{Optical design performance}

Davies-Cotton telescopes are equipped with a single reflector which is constructed of many identical mirror facets. The facets are typically hexagonal in shape and are sections of a spherical surface with focal length F. These are arranged on a mirror cell (the dish) having a spherical profile with radius $\mathrm{F}$. This design has been adapted from solar concentrators [3]. For F/D values around 1.5, this leads to optical performance that is somewhat better than that of an equivalent parabolic reflector for off-axis incoming rays and, for telescopes of the size of the SST, is acceptable for field angles of up to a few degrees. This is illustrated in Figure 3, which shows the $80 \%$ encircled energy diameter as a function of the field angle.

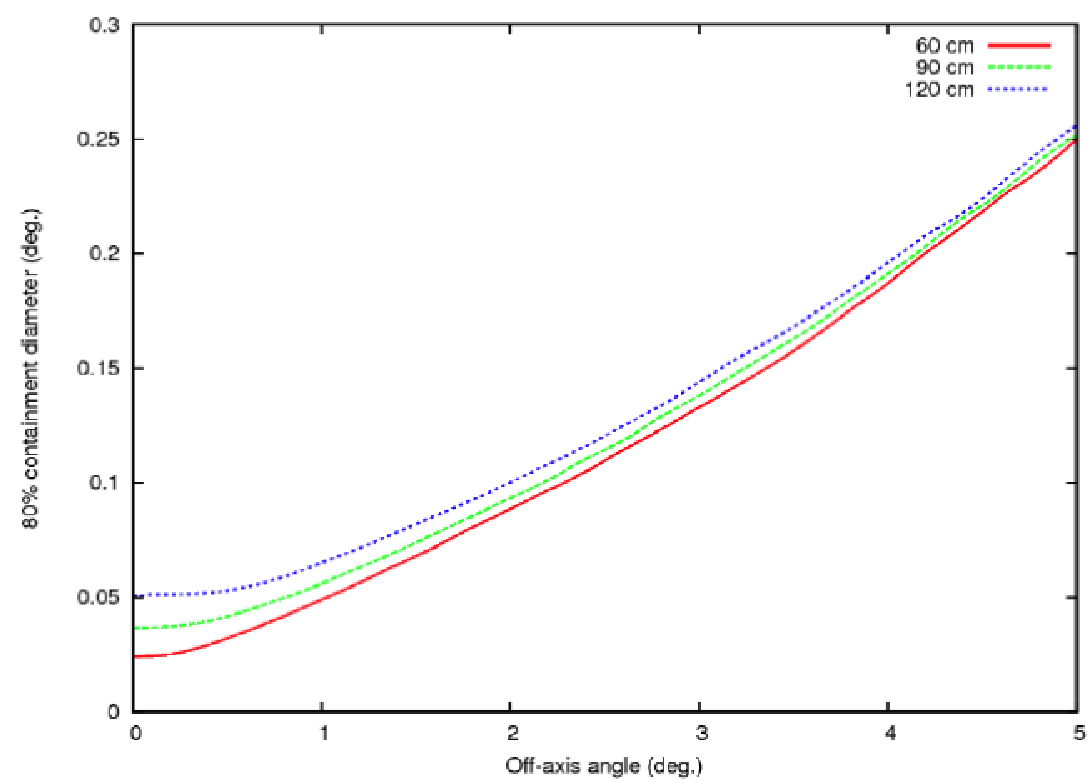

Figure 3. The $80 \%$ containment diameter for a Davies-Cotton telescope with $\mathrm{F}=11 \mathrm{~m}$ and $\mathrm{D}=7 \mathrm{~m}$ and hexagonal mirror segments of sizes (flat-to-flat) 60, 90 and $120 \mathrm{~cm}$. 


\subsection{Mechanical structure designs}

The Institute of Nuclear Physics (IFJ) of the Polish Academy of Sciences (PAN) is designing a telescope of the above type, consisting of a mast, a dish and dish support structure, as well as the columns which support the telescope. The mast positions the camera with respect to the mirrors, which are mounted on the dish, which in turn is attached to the dish support structure (see Figure 4). The rigidity of the mast is increased by the use of pre-tensioned steel rods. The dish structure is mounted on the telescope support columns. All steel profiles and tubes can be obtained as off-the-shelf products from industry.

The dish is built of square steel profiles with increased thickness in areas where the dish is connected to the dish support. It is a welded structure of about $6 \mathrm{~m}$ in diameter composed of two identical, symmetric parts which are bolted together. It accommodates 36 hexagonal mirror tiles of size $1.05 \mathrm{~m}$ (flat-to-flat). In a similar way, its support structure is welded out of square steel profiles with the wall thickness increased in parts of the structure in which the toothed-rack of the elevation drive system, the main axis, and the mast are installed. It can be split into two symmetric parts for easy transportation. The number of profiles was minimized to simplify the design and to reduce the cost. The back structure supports a 4 ton counter-weight. Note that the mast is attached directly to the dish-support structure, not the dish, so that no stress is transferred directly to the dish. The design of the mast guarantees the proper location of the camera with respect to the reflecting surface. The mast is built of circular steel tubes and steel rods pre-stressed with a force of $3500 \mathrm{~N}$ by means of turnbuckles.

Two vertical columns support the elevation axis that allows rotation from the parking position at $-13^{\circ}$ up to $95^{\circ}$. The columns are built of steel square profiles and bolted to the rotating platform. A crown roller bearing with diameter of $2 \mathrm{~m}$ is bolted to the base of the rotating platform and to the concrete foundation and allows for rotation of the telescope in azimuth $\left( \pm 270^{\circ}\right)$.
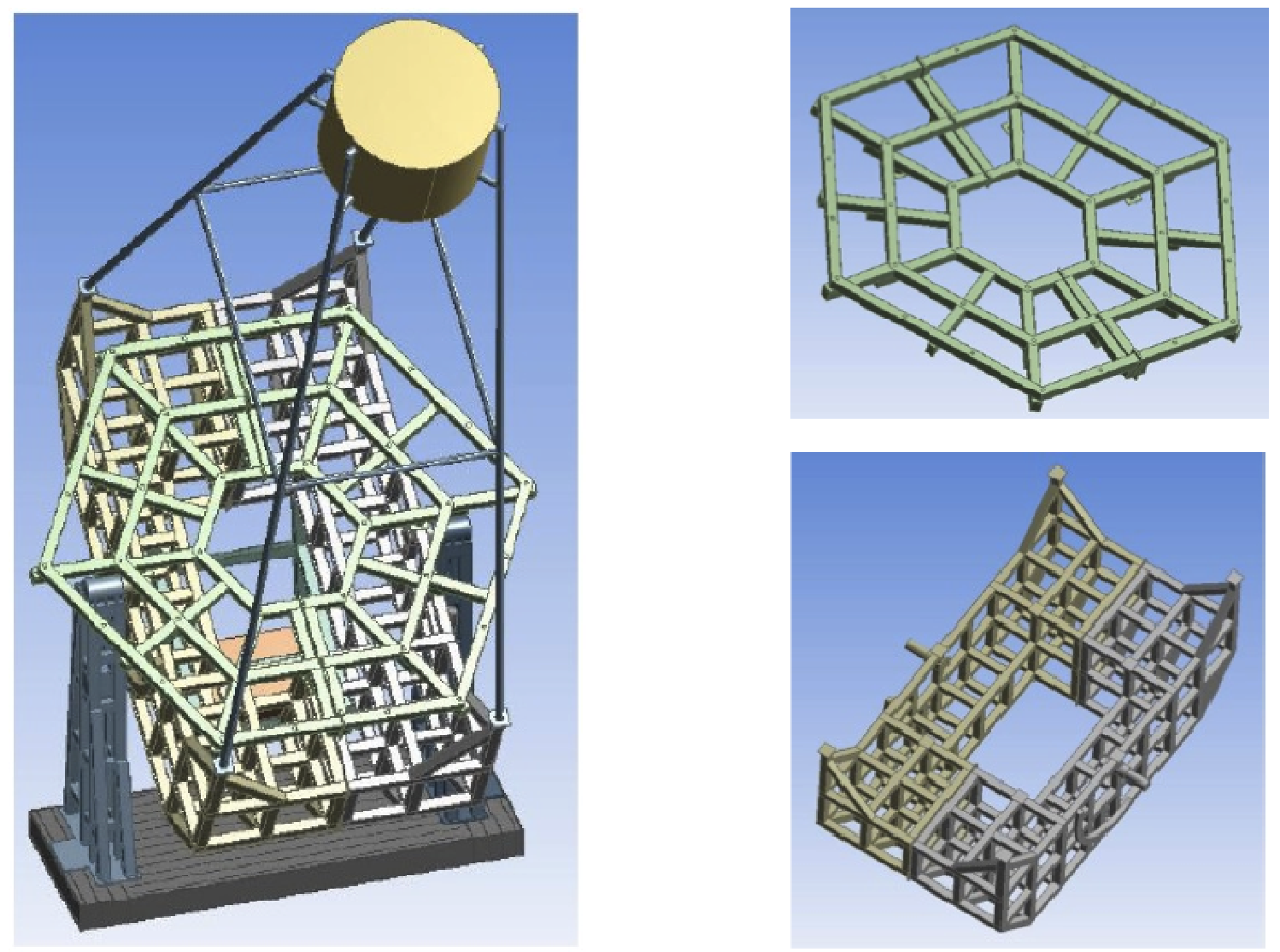

Figure 4. (Left panel) Illustration of the SST-DC telescope structure. The camera, mast, dish, dish-support structure, supporting columns and platform are shown. (Top-right panel) Detail of the dish structure. (Bottom-right panel) Detail of the disc-support structure. 
Finite Element Analysis (FEA) has been used to guide the design and to perform structural checks. The main loads applied on the structure include its dead weight (including camera), the wind (for operative and survival conditions), ice and snow. Studies so far have confirmed that the structure meets the requirements for the observations and is able to withstand the maximum survival wind speed of $200 \mathrm{~km} / \mathrm{h}$. The eigenfrequencies at which the structure oscillates have also been calculated at various angles of elevation. At a telescope elevation of $60^{\circ}$, the lowest value obtained is $2.2 \mathrm{~Hz}$; Figure 5 shows the three lowest oscillation modes.
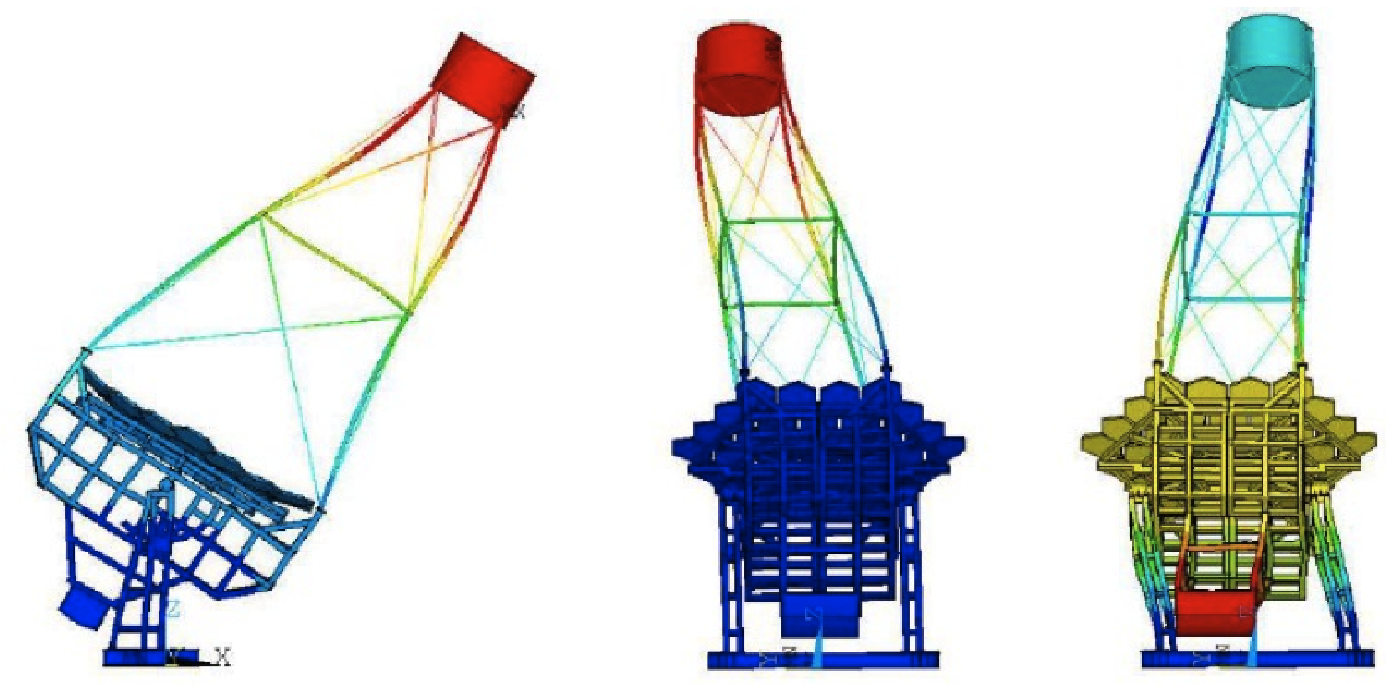

Figure 5. The three lowest frequency eigenmodes, (i), (ii) and (iii) from left to right, of the oscillations of the SST-DC structure.

The elevation drive system is composed of two $1.5 \mathrm{~m}$ radius-of-curvature toothed-racks (off-the-shelf products), each driven by an electric motor of power about $8 \mathrm{~kW}$. The motors are fixed to the telescope support columns and provide backlash free steering of the telescope's elevation. This drive system will be equipped with 4 encoders ( 2 in the motors and 2 independent devices on the axis). The steering program will check the consistency of readouts from all encoders. The system will also be equipped with end-switches on each side. There will be one brake per side. The azimuth drive system is composed of a roller crown bearing driven by two motors of about $7 \mathrm{~kW}$ power. The system will operate in two modes. In the first mode both motors work in the same direction. This mode will be used for quick movements of the telescope during the positioning/parking phase. In the second mode, the motors work in opposition in order to avoid backlash. This mode will be used for tracking. The azimuth drive system will be equipped with end-switches equivalent to those used for the elevation drive. There will be one brake fixed to the crown bearing.

\subsection{Mirror design}

The radius of curvature of the hexagonal mirror segments required for the SST-DC is approximately $23 \mathrm{~m}$ and their size is $1.05 \mathrm{~m}$ flat-to-flat. These are being developed by IFJ PAN using glass cold slumping technology, developed at the INAF-Brera Astronomical Observatory [4][5], and an open structure composite mirror design. FEA studies have demonstrated that it may be difficult to achieve the required radius of curvature using the cold slumping technique: the 
stresses induced in the glass sheet as it is moulded are potentially too high and cause the mirror to have a short lifetime. Alternatives are therefore under consideration.

\subsection{Camera designs}

The attraction of the SST-DC option is not only the wealth of experience with Davies-Cotton telescopes that exists within past and present IACTs experiments but also the fact that, for appropriately chosen telescope parameters, the cameras of the SST-DC and the MST could be similar, leading to some economies of scale. In particular, we choose to use the same 1.5" PMTs for the MST and SST, giving pixels of physical diameter $\mathrm{d}=50 \mathrm{~mm}$. The required angular pixel pitch for the SST is $0.25^{\circ}$. Assuming a telescope field-of-view of $10^{\circ}$, the camera should host about 1459 pixels within a diameter of about $2.05 \mathrm{~m}$ and have a mass of about $1600 \mathrm{~kg}$. More details on the developments on going for the MST camera can be found in [6][7].

\section{DUAL-MIRROR TELESCOPES: THE SCHWARZSCHILD-COUDER DESIGN}

We are also investigating the use of dual mirror SSTs (SST-DM). As has previously been demonstrated [8], dual mirror Schwarzschild-Couder telescopes allow better correction of aberrations at large field angles and hence the construction of telescopes with a smaller focal ratio. This implies that, for a given primary mirror and angular pixel size, the physical pixels are smaller. The cameras for these telescopes can thus be based on multi-anode PMTs (MAPMTs) or Silicon PMs (SiPMs) and can be considerably cheaper than those envisioned for the SST-DC. The SST group within CTA has designed dual-mirror telescopes which have the potential to provide the required optical performance and allow exploitation of these technologies. For similar reasons, groups working on the development of the MST are also pursuing this option [9].

The Italian ASTRI (Astrofisica con Specchi a Tecnologia Replicante Italiana) project run by INAF (Istituto Nazionale di AstroFisica) is producing a complete design of a SST-DM [10]. Rapid progress is being made by this group towards the construction of an end-to-end prototype telescope composed of the telescope structure itself, the mirrors, the camera based upon SiPMs and the control software.

A second telescope is being prototyped in France as part of the GATE (GAmma-ray Telescope Elements) project [11]. It is based on the optical design produced in the UK in Durham, as was the preliminary mechanical design. UK, US and Japanese groups within CTA are designing a further camera for the dual mirror SST, the Compact High-Energy Camera (CHEC), based on MAPMTs.

In the following, the optics and mechanical structure of the ASTRI and GATE telescopes are described. A further section focuses on the two cameras. Only the telescope and camera that will give the best performances versus cost will then be adopted for the final implementation of the CTA-SST array.

\subsection{Optical design performance}

Matching the physical size of the pixels offered by MAPMTs or SiPMs sensors (a few millimetres) to the required angular pixel size of the SST implies that the focal length of the telescope $\mathrm{F} \sim 2 \mathrm{~m}$. Ensuring sufficient collection area to obtain efficient triggering in the SST energy range, that is, a primary mirror of diameter about $4 \mathrm{~m}$, then requires that the telescope's focal ratio be about 0.5 .

The proposed designs have the Schwarzschild-Couder configuration, optimized using the commercial software ZEMAX, ensuring a light concentration higher than $80 \%$ within the dimension of the pixels over a wide field. The mirrors profiles are aspheric with substantial deviations from the main spherical component (see Figure 6 bottom panels). The ASTRI 
and UK designs somewhat differ a bit one to each other; hereinafter we describe the ASTRI design as a reference. This design has been optimized taking into account also a realistic way to implement the telescope, such as the segmentation of the primary mirror M1 and the arrangement of detection units into the detector. The optical system is shown in the top left panel of Figure 6. It has a plate scale of $37.5 \mathrm{~mm} /{ }^{\circ}$, a pixel size of approximately $0.17^{\circ}$ and an equivalent focal length of $2150 \mathrm{~mm}$. This setup delivers a corrected field of view up to $9.6^{\circ}$ in diameter as shown by the enclosed energy curves plotted in Figure 6 (top right panel). Concerning the throughput, a mean value of the effective area of about 6.5 $\mathrm{m}^{2}$ is achieved, taking into account: the segmentation of the primary mirror, the obscuration of the secondary mirror, the obscuration of the detector, the reflectivity of the optical surfaces as a function of the energy and incident angle, the losses due to the detector's protection window and finally the efficiency of the detector as a function of the incident angles (ranging from $25^{\circ}$ to $72^{\circ}$ ). In Table 1 we summarize the geometry of the optical system: the resulting telescope is compact, having an $\mathrm{M} 1$ diameter of $4 \mathrm{~m}$ and a primary-to-secondary distance of $3 \mathrm{~m}$.
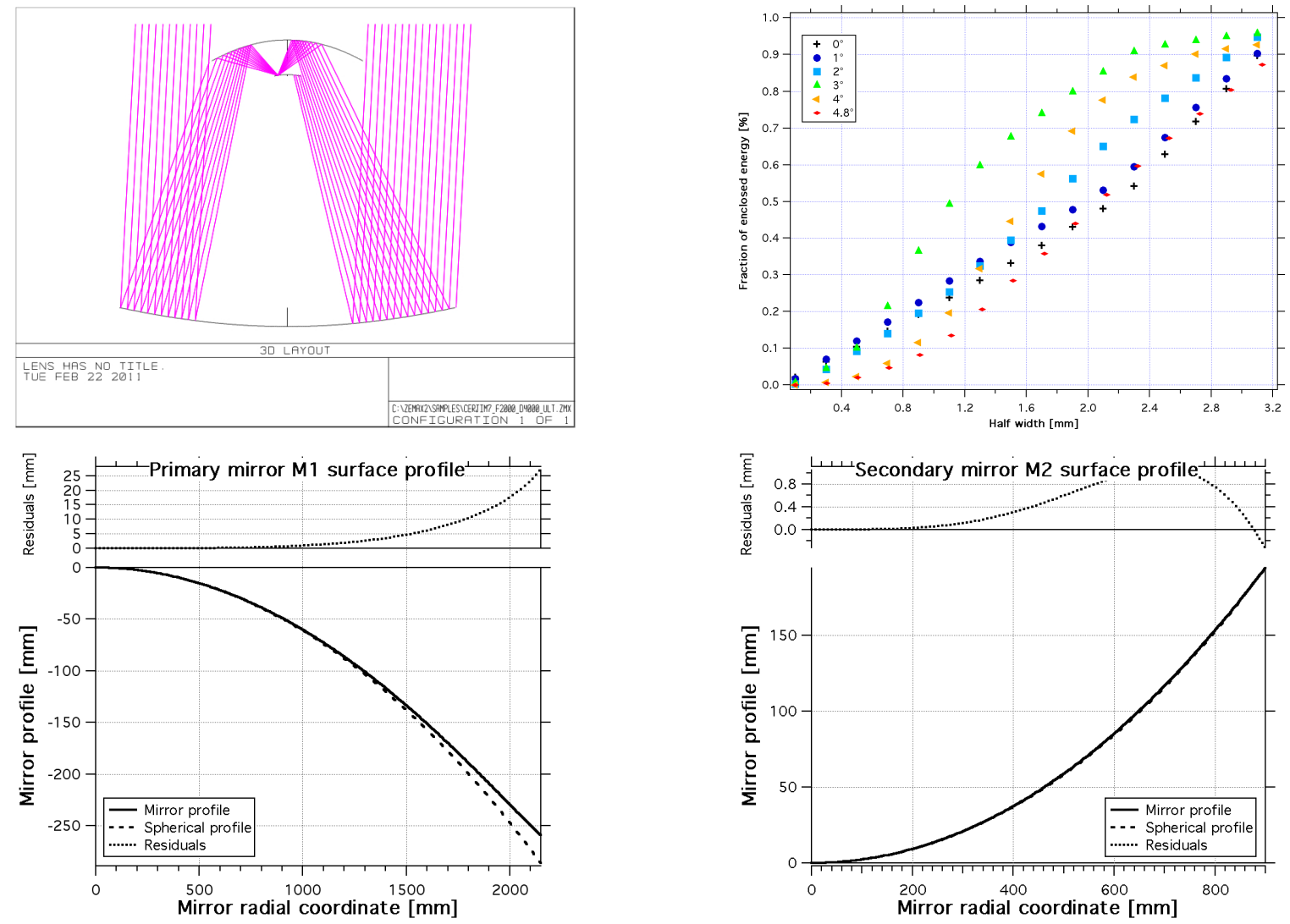

Figure 6. (Top-left panel) the Schwarzschild-Couder optical layout adopted for the ASTRI end-to-end prototype. (Top-right panel) the fraction of the energy from a point source contained within a square as a function of the half-width of the square for various field angles. (Bottom panels) radial profiles of the primary M1 (left) and secondary M2 (right) mirrors, and deviations from sphere.

Table 1. Main geometrical dimensions of the ASTRI optical system.

\begin{tabular}{|l|r|r|r|r|}
\hline ELEMENT NAME & $\begin{array}{c}\text { DIAMETER } \\
{[\mathbf{m m}]}\end{array}$ & $\begin{array}{c}\text { RADIUS OF CURVATURE } \\
{[\mathbf{m m}]}\end{array}$ & SHAPE & $\begin{array}{c}\text { DISTANCE TO... } \\
{[\mathbf{m m}]}\end{array}$ \\
\hline M1 & 4306 & -8223 & Even asphere & M2: 3108.4 \\
\hline M2 & 1800 & 2180 & Even asphere & DET: 519.6 \\
\hline DET & (side) 360 & 1000 & -- & -- \\
\hline
\end{tabular}




\subsection{Mechanical structures designs}

Both the ASTRI and GATE telescopes adopt an alt-azimuthal design in which the azimuth axis will permit a rotation range of $\pm 270^{\circ}$. The mirror cell is mounted on the azimuth fork which allows rotation around the elevation axis from $-5^{\circ}$ to $+95^{\circ}$. Fixed on the mirror cell is the mast structure that supports the secondary mirror and the camera. The mast has a quadrupod layout, but with different solutions between the two projects. In order to balance the torque due to the overhang of the optical tube assembly with respect to the horizontal rotation axis, two counterweights are also supported from the mirror cell. Figure 7 shows the overall assemblies of ASTRI and GATE.
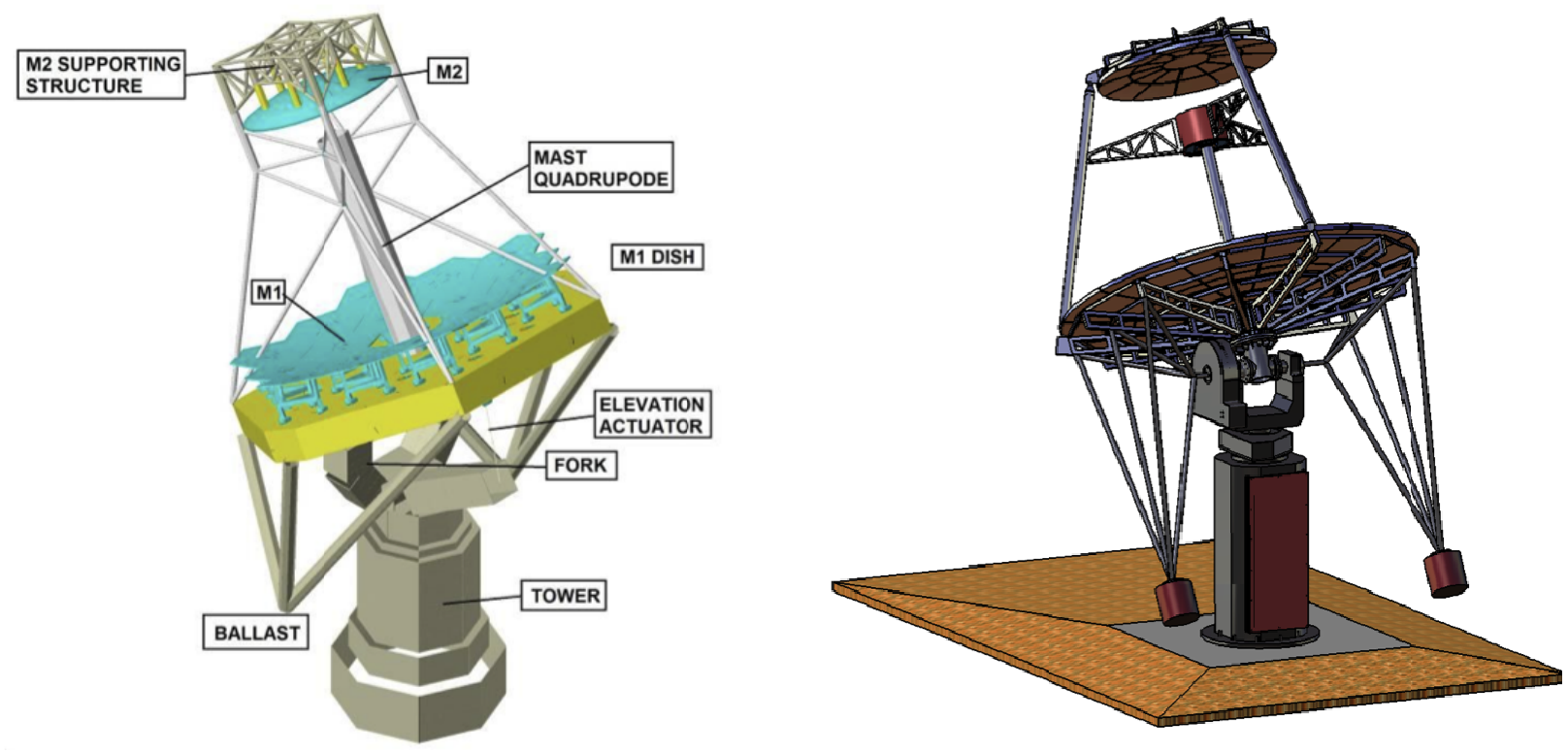

Figure 7. (Left panel) The ASTRI telescope structure showing the tower and fork, the counterweights, the M1 and M2 mirror support structures, the quadrupod and its central tube and the elevation actuator. (Right panel) The GATE telescope structure, shown here is the version with a shallow fork.

\section{The ASTRI design}

In the ASTRI design, the dish is a ribbed steel plate of about $40 \mathrm{~cm}$ thickness constructed in two halves to allow easy transportation. To this are attached the supports for the mirror segments, each of which includes a single and a double axis actuator and a bearing, which allows steering of the segments for alignment purposes. The quadrupod legs, with their radial bracing, counteract the lateral deformations of the mast structure due to gravitational and wind loads, while the central tube increases the torsional stiffness of the structure. Attached to the foundations is the tower, which ensures that the remaining telescope structure is at the correct height above the ground. At the top, it is interfaced to the azimuth fork. From the structural point of view the tower transmits loads (vertical and lateral) from the azimuth fork to the foundation. It is envisaged that some mechanical and electrical elements of the telescope equipment will be located in the space in the tower, so openings to access this equipment are foreseen. The tower will consist mainly of a steel tube, with section variations along its height. At the top of the tower is attached the azimuth fork, the interface with the tower being the azimuth bearing. The fork supports the elevation assembly (dish, counterweight and quadrupod) through the linear actuators and the bearings at its upper ends. The fork transmits loads coming from the elevation assembly to the tower and is composed of welded steel box sections. All steel structures are protected against corrosion by paint. The dish structure is attached to the azimuth fork using two preloaded tapered roller bearings, one on each of the fork's arms. At the upper end of the mast is the structure for supporting the monolithic secondary mirror. This consists of three actuators attached to the mirror via whiffletrees to ensure the load is spread over a sufficiently large area. In addition, three lateral 
arms support the transverse components of the secondary mirror's weight as they vary with the orientation of the telescope.

The azimuth drive is located at the base of the tower and is composed of two pinions, driven by electric motors, that couple with a rim gear. Axially pre-loaded ball bearings complete the azimuth assembly. The linear actuator, which drives the elevation, attaches to the tower at its lower and the dish structure at its upper end. The actuator consists of a preloaded ball screw driven through a gearbox by an electric motor. The orientation of the telescope is determined using absolute encoders located on each of the azimuth and altitude axes. Safety is ensured during movement by a system of software and electro-mechanical switches.

Also in this case, FEA has been used to evaluate the performance of the system. The lowest frequency eigenmode of the oscillations of the dish and mast is $8.5 \mathrm{~Hz}$. FEA has also been carried out to determine the effects on the telescope of temperature gradients. This indicates that temperature differences of $\pm 1^{\circ} \mathrm{C}$ from the base of the mast and its central tube to the top of the mast are inconsequential. However, similar temperature shifts across the dish support structure can lead to significant contributions to the misalignment of the primary mirror segments. Temperature variations in the tower structure can also lead to noticeable effects on the telescope pointing. Studies to understand the likely magnitude of these effects in operation are underway, as are strategies for dealing with any residual problems in these areas.

\section{The GATE design}

The main elements of the GATE structure are, again, the foundation on which the tower/fork structure is mounted via the azimuth drive system. The version shown has a shallow fork structure for which two counter weights are needed, mounted exterior to the fork. Also under study is a design with a shorter tower and a deeper fork, allowing a single counterweight to be mounted centrally. In both cases, the fork is fastened via the azimuth bearings and drive to the primary dish structure, which carries the secondary mirror support and the camera. Aluminum and carbon fiber have also been considered, but finally not selected to optimize costs and easiness of manufacturing and mounting.

The drive systems under study consist either of crown gears which are driven by pinions connected to electric motors via a gearbox, or worm gears, again driven by electric motors.

FEA has determined that the lowest frequency eigenmodes of the oscillations of the telescope involve transverse motion of the secondary with respect to the primary and are about $5 \mathrm{~Hz}$. Rotational eigenmodes of oscillation have lowest frequencies of about $12 \mathrm{~Hz}$. These values refer to a preliminary design not yet optimized.

\subsection{Mirror designs}

For the ASTRI telescope it is proposed to construct the primary mirror as a set of 18 hexagonal-shaped panels having $850 \mathrm{~mm}$ face-to-face dimension. Three different types of mirror profiles are necessary to reproduce the M1 profile. GATE will exploit petal-shaped segments. The number and dimension of these is still under investigation. It is hoped that only two types of segment will be required. For both telescopes, the aim is to build a monolithic secondary mirror. More details about the technology under development can be found in [12].

\subsection{Camera designs}

\section{The ASTRI camera based on SiPM sensors}

The ASTRI camera design uses SiPMs as photosensors. The pixels contain 3600 cells, each of which is an avalanche photodiode operated in quenched Geiger mode. These cells are of dimensions $50 \times 50 \mu \mathrm{m}^{2}$, giving a fill factor of $70 \%$. The particular device chosen is the Hamamatsu S11828-334 monolithic multi-pixel SiPM consisting of 4x4 pixels of roughly $3 \times 3 \mathrm{~mm}^{2}$. Four of these are grouped together to form one pixel of physical size $6.2 \times 6.2 \mathrm{~mm}^{2}$, matching the required angular size. As is shown in the top left panel of Figure 8, four of the Hamamatsu devices are put together to 
form a unit. Four such units then form a module called a Photon Detection Module (PDM - shown in the top right panel of the same figure). This module is composed of 16 Hamamatsu devices and has dimensions of 56x56 $\mathrm{mm}^{2}$. The PDMs are constructed by plugging the Hamamatsu devices into connectors attached to a printed circuit board (PCB). Figure 8 also shows one unit attached to a PCB on which the connectors for the remaining 15 units are visible (bottom left panel). Under each unit on the PCB is a small temperature sensor, allowing the temperature of the SiPMs to be monitored, providing a route through which the temperature dependent SiPM gain can be stabilised. A schematic layout for the Front End Electronics (FEE) is aslo shown in the remaining panel of Figure 8. The FEE boards supply the power for the SiPMs, perform the readout and form the first trigger signals. Several Application Specific Integrated Circuits (ASICs) are available for the readout of the camera. Under consideration are in particular the TARGET series of chips and the EASIROC (Extended Analogue SiPM Readout Chip). A range of simulation tools has been produced to aid the design of the readout and trigger. Moreover, as visible from the same figure, there are small gaps between the Hamamatsu devices when they are mounted to form units and PDMs. In order to recover the light that would be lost in these gaps, light guides are placed over them. These are of thickness $2.5 \mathrm{~mm}$ and made of high refractive index glass; these are designed to utilize the total reflection occurring at the light guide's side walls.
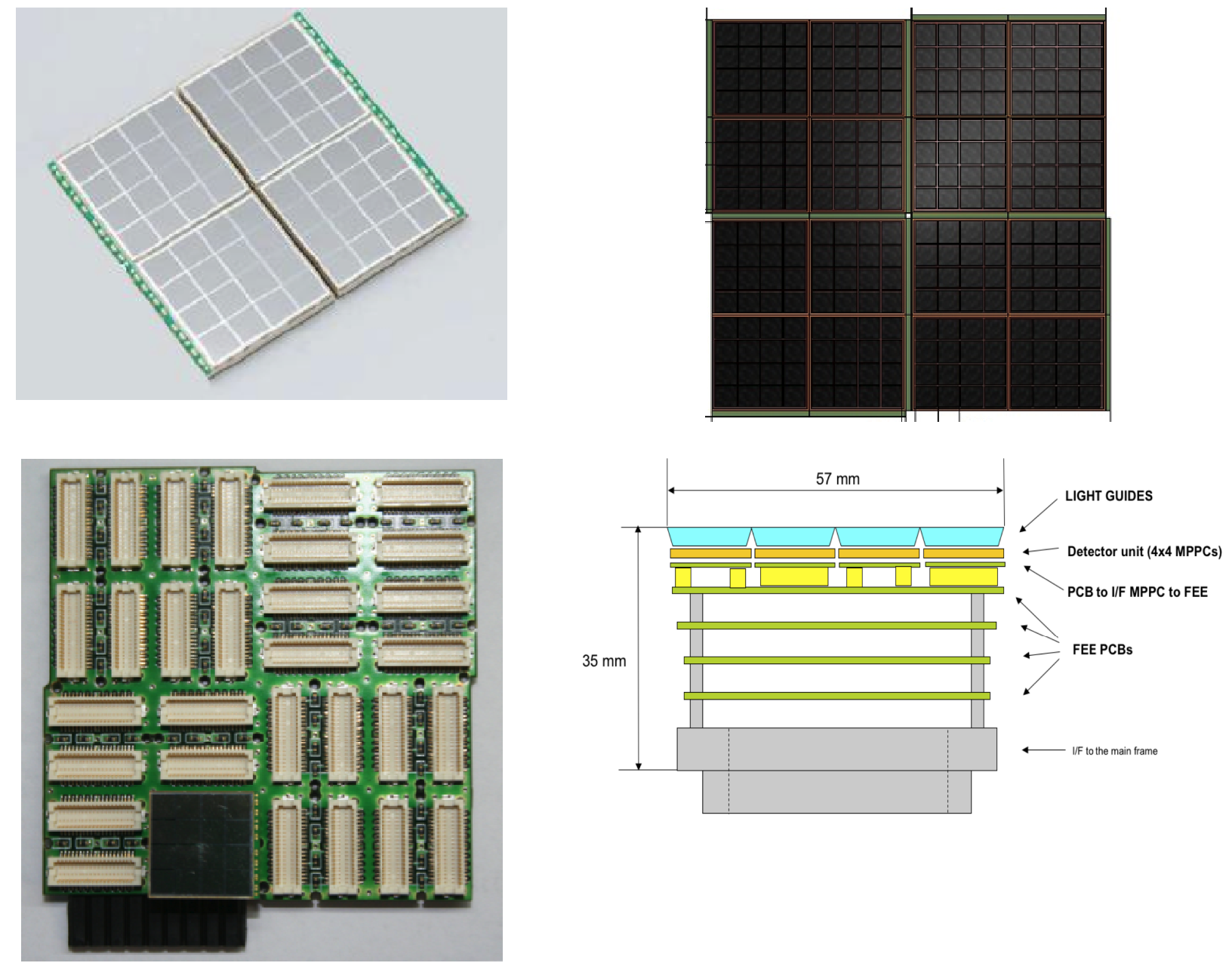

Figure 8. (Top-left panel) Four Hamamatsu S11828-334 SiPMs form a detector unit (Top-right panel) and four such units a PDM. (Bottom-left) The PCB on which the connectors for the Hamamatsu units are soldered; one Hamamatsu device is plugged into its socket in the bottom left of the picture. (Bottom-right panel) Support structure for the FEE of one PDM unit, on top of each SiPM the light guides are visible. 
In addition to the FEE mentioned above, design of the back end electronics is underway. This will use a Field Programmable Gate Array (FPGA) and local memory to provide interfaces to the CTA data acquisition, to the camera controller and to the CTA clock. There will also be circuitry to provide the various DC voltages needed to power the elements of the camera.

A schematic diagram showing the location of the module and the various electronics boards is shown in Figure 9 (left panel). This also gives the dimensions of the camera. The total height of the camera is about $30 \mathrm{~cm}$. A preliminary design of the chassis of the complete camera is also shown (right panel). The camera lid can be seen in this picture, this can be closed to protect the sensors from the elements. As the focal plane of the dual mirror telescopes is convex, with a radius of curvature of $1 \mathrm{~m}$, the PDMs must be attached to a precisely machined curved plate. Below the sensor plane is the support structure to which further electronics boards and the cooling system can be attached.
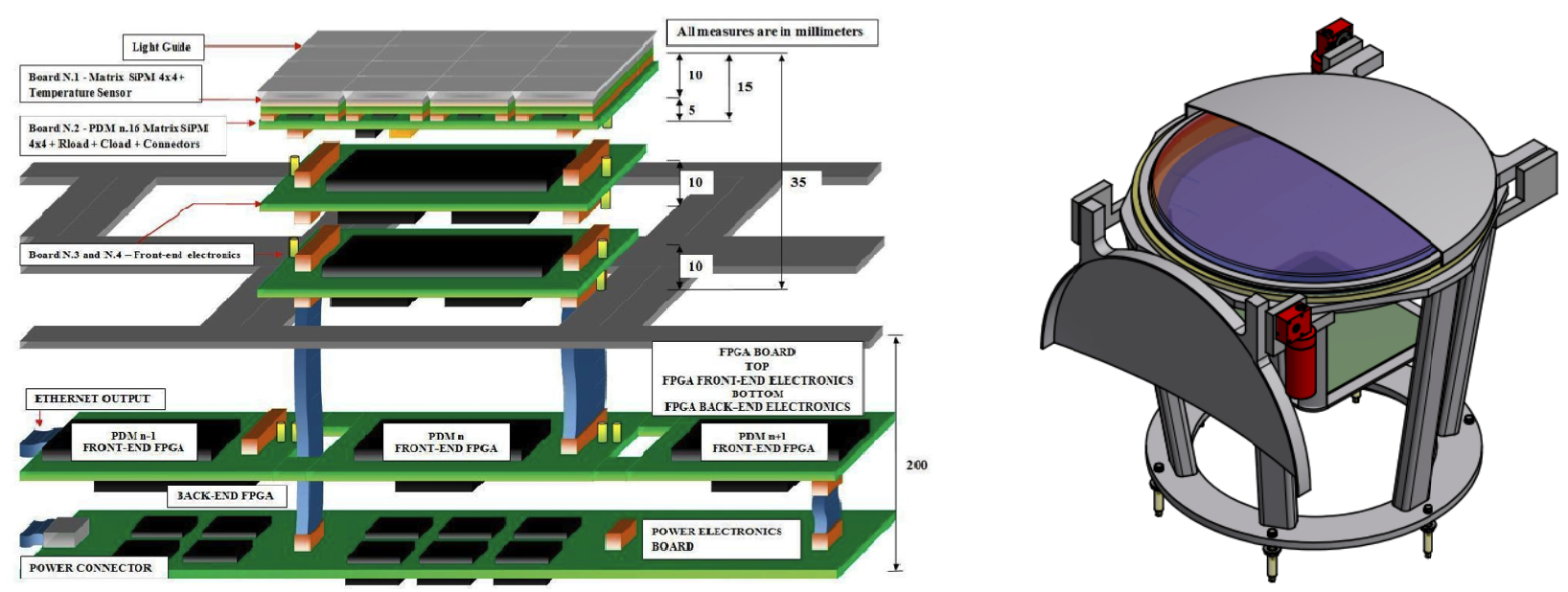

Figure 9. (Left panel) Schematic diagram of the camera electronics for the ASTRI telescope. (Right panel) The preliminary layout of the camera chassis.

\section{The CHEC camera based on MAPMTs sensors}

The default photosensor for the Compact High-Energy Camera is the Hamamatsu H10966 MAPMT. This consists of 64 pixels of size $6 \times 6 \mathrm{~mm}^{2}$, in a unit of dimensions $52 \times 52 \mathrm{~mm}^{2}$. Thirty-two MAPMTs can be used to cover the focal plane, providing a field of view of about $9^{\circ}$.

Each FEE module provides the high voltage supply needed by a MAPMT, samples the signals produced by its 64 channels at a frequency of about $500 \mathrm{MHz}$, forms a first level trigger by applying thresholds to sums of four pixels and outputs a digitized waveform for each of the MAPMT's channels. CHEC will make use of modules designed at SLAC for the Advanced Gamma-ray Imaging System (AGIS) based on the TARGET ASIC (see Figure 10 top panel) [13]. Minor modifications to this are needed and these will be carried out in collaboration with SLAC. Unfortunately, the TARGET modules, as they are, cannot be connected directly to the MAPMTs in the CHEC as the curvature of the focal plane would then require large gaps between the MAPMTs. The MAPMT is instead attached to a preamplifier board and then a structure containing a twisted length of ribbon cable, allowing bending in two planes, which carries the signals from the MAPMT to the electronics. The preamplifier board behind the MAPMT allows the PMTs to be operated at low gain, important given the high counting rate they will experience due to background photons. The preamplifier also allows shaping of the MAPMT signal and hence optimization of the performance of the FEE. Further, if it becomes clear 
that SiPMs will offer better performance per unit cost than MAPMTs, the MAPMT photosensor plane with its preamplifiers can be replaced with a SiPM-based system with new preamplifiers ensuring the correct signal shape enters the TARGET module. A mechanical frame that provides the required rigid support has been designed. This system is illustrated in Figure 10 (bottom panel). Prototype mechanical structures have shown that this system functions as hoped, allowing the MAPMTs to be placed on a curved surface.
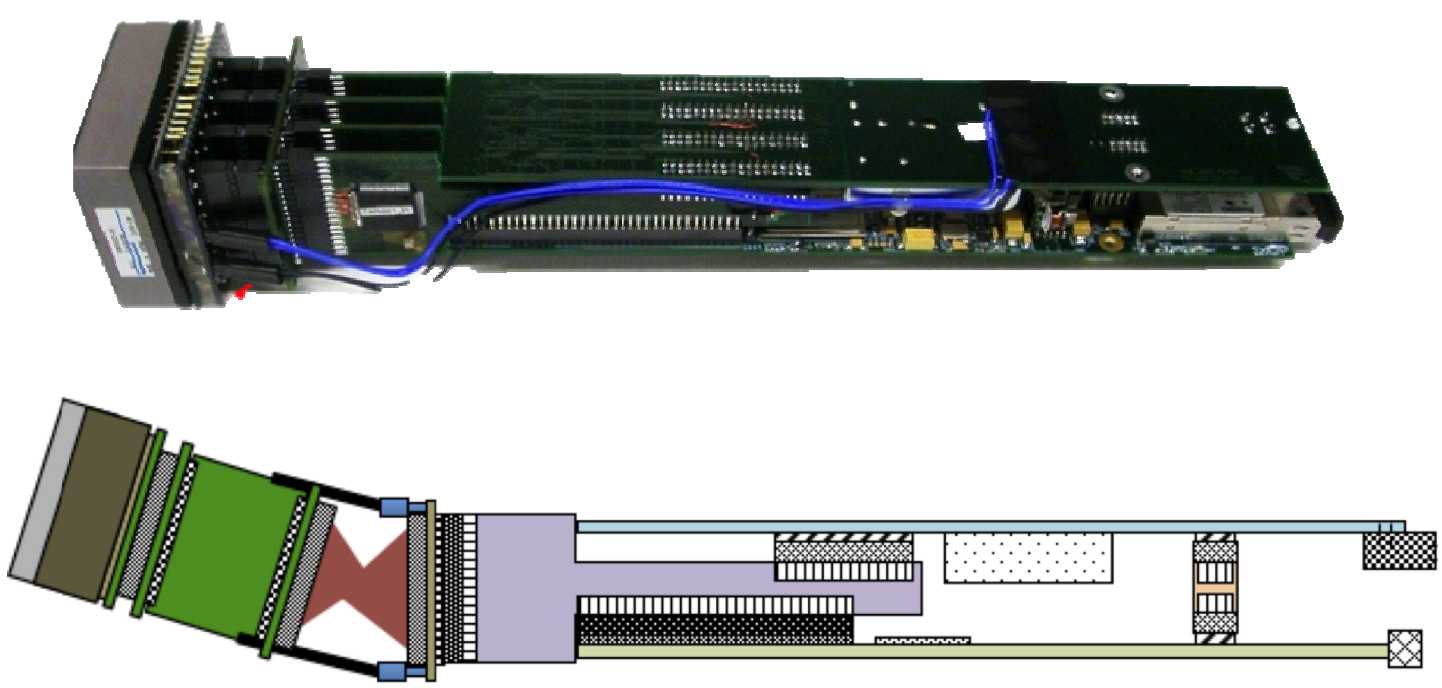

Figure 10. (Top panel) A TARGET module attached to a Hamamatsu MAPMT; note that the direct attachment shown here is replaced by a flexible system in the CHEC, as is described in the text. (Bottom panel) Illustration of the mounting of a MAPMT on a TARGET module, showing the bending allowed by the structure.

The trigger signals provided by the FEE modules must be combined and examined to select candidate Cherenkov events against the night-sky background. This camera trigger system forms part of the Back-End Electronics (BEE), which is also responsible for processing data from the FEE modules and distributing clock signals with the required level of precision to the FEE modules. Recent developments mean that it is feasible to process trigger signals with nanosecond accuracy and sub-nanosecond delay correction from all FEE modules in one or two FPGAs. The entire camera trigger can be performed on a high-density, active backplane, housing the FPGAs and connected directly to the FEE modules. The backplane also routes the serial data from the FEE modules to a single location for processing. The read-out electronics may be placed on a mezzanine card, and use a dedicated FPGA to compress the data for transmission to a central array location, or on an embedded PC located at the rear of the camera and connected to the BEE trigger backplane via Ethernet. An active backplane is under development by CTA collaborators at Washington University; the BEE design will be developed with this team.

The mechanical aspects of the camera include a support matrix for the MAPMTs, internal elements for support of the electronics, a cooling system and an external structure, which includes a lid and the interface to the telescope. The MAPMT support matrix must ensure precise focal-plane positioning. The internal structure must allow adequate cooling of the electronics and remain stable on decade timescales to repeated camera movements. Thermal modelling of the camera will be done during the camera design to assess the cooling and control requirements, which will be implemented during the mechanical prototyping. The external structure must be weather-proof and minimise dust ingress, and provide minimal additional shadowing of the primary mirror. The most complex element of the external structure is the lid, which must be remotely operated, highly reliable and provides a screen for the imaging of stars for alignment purposes. 
Each aspect must be designed with assembly and access procedures in mind. Figure 11 shows a schematic diagram of the CHEC.

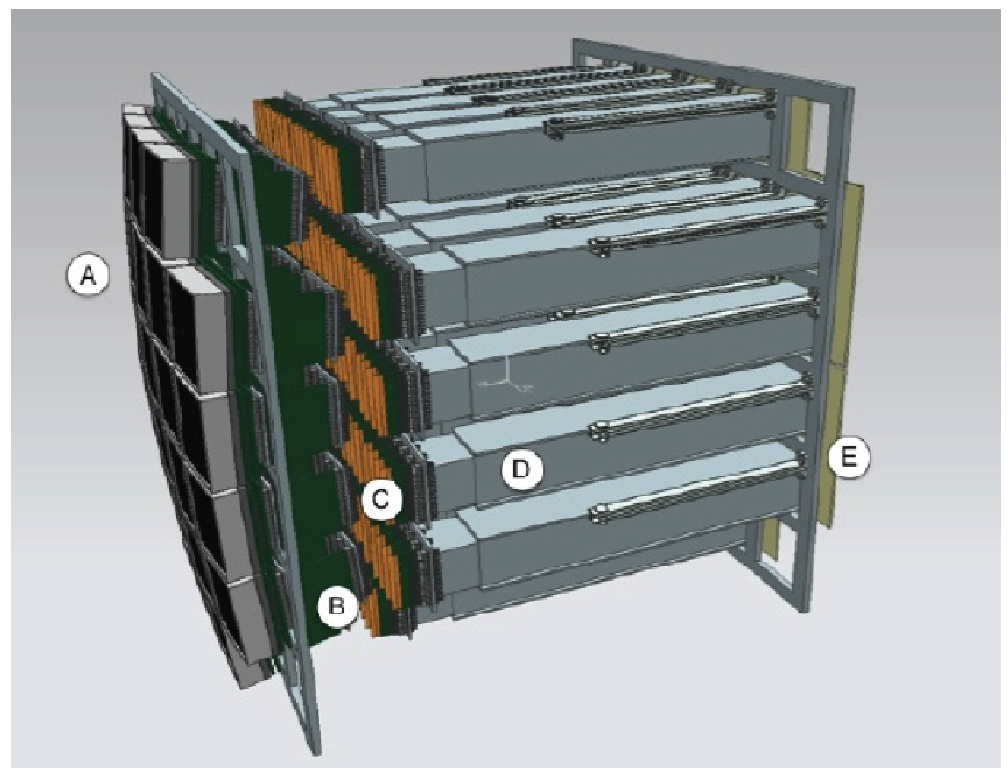

Figure 11 Conceptual design of the CHEC showing: (A) MAPMTs, (B) preamplifier boards, (C) interface to FEE modules in which the curvature of the focal plane is removed, (D) SLAC TARGET-based FEE, (E) back-plane, which will include BEE; the MAPMT position matrix, external mechanics, power-supplies, cooling system and control computer are not shown.

\section{CONCLUSIONS}

The SST array is the section of CTA which dominates the sensitivity of the instrument in the high photon energy regime. The area covered by the SST array therefore determines the highest energies to which CTA is sensitive and the quality of the measurement of the highest energy photons. This is determined by the quality of the individual telescope images, but also crucially by the number of telescopes observing the showers caused by those photons. Both the area the SST array can cover and the multiplicity of the measurements it makes increase if more telescopes can be built, making it important to reduce the cost of the telescopes to the lowest possible level. Hence, although the SST array can be built using the Davies-Cotton telescopes with PMT-based cameras that have traditionally been used for IACTs and will be used for the LST and the MST, this may not represent the best option for CTA. In parallel with the design of a SST-DC telescope, the SST group is therefore investigating alternative designs. The dual mirror telescope allows the reduction of the plate scale to levels that make it possible to use SiPM arrays or MAPMTs in a compact camera. Compared to the conventional SSTDC design, this reduces the camera cost enormously, at the expense of introducing a more complex optical system, with its attendant costs. If the group is successful in producing functioning dual mirror telescopes, the indications are that these will be cheaper and will represent a better option.

The potential benefits of the alternatives to the SST-DC make it important that these are investigated fully: the result may be a significantly improved CTA instrument. The risks and uncertainties associated with these novel designs mean that a conventional telescope of the required size must also be constructed. This implies that the SST group must continue to pursue all the above options in parallel, focusing its efforts on the telescopes offering the best performance per unit cost as and when it becomes clear these function as hoped and the costs are as expected. 


\section{REFERENCES}

[1] Weekes, T. C., et al., "Observation of $\mathrm{TeV}$ gamma rays from the Crab nebula using the atmospheric Cerenkov imaging technique", The Astrophysical Journal, 342:379\{395\}, July 1989.

[2] Actis, M. for the CTA consortium, "Design concepts for the Cherenkov Telescope Array CTA: an advanced facility for ground-based high-energy gamma-ray astronomy", Experimental Astronomy, volume 32, pages 193-316, (2011)

[3] Davies, J. M., Cotton, E. S., "Design of the Quartermaster Solar Furnace", Solar Energy Sci. Eng. 1 (1957) 16-22.

[4] Pareschi, G., et al., "Glass mirrors by cold slumping to cover $100 \mathrm{~m}^{2}$ of the MAGIC II Cerenkov telescope reflecting surface”. Proc. SPIE 7018, (2008).

[5] Vernani, D., et al., "Development of cold-slumping glass mirrors for imaging Cerenkov telescopes". Proc. SPIE 7018 (2008).

[6] Kubo H. for the CTA consortium, "Development of the Readout System for CTA Using the DRS4 Waveform Digitizing Chip", $32^{\text {nd }}$ ICRC (2011).

[7] Puehlhofer G. for the CTA consortium, "FlashCam: A camera concept and design for the Cherenkov Telescope Array", $32^{\text {nd }}$ ICRC (2011).

[8] Vassiliev, V. V., Fegan, S. J., Brousseau, P. F., "Wide field aplanatic two-mirror telescopes for ground-based gamma-ray astronomy", Astropart. Phys, 2007, vol. 28, issue 1, p. 10-27.

[9] Cameron, R. A., et al., "Development of a mid-sized Schwarzschild-Couder Telescope for the Cherenkov Telescope Array". Proc. SPIE, 8444-43 (this conference).

[10] Canestrari, R. for the ASTRI collaboration, "The Italian ASTRI program: an end-to-end dual-mirror telescope prototype for Cherenkov light imaging above few TeV", $32^{\text {nd }}$ ICRC (2011).

[11] Laporte, P., et al., "An Innovative Telescope for the Very High Energy Astronomy". Proc. SPIE, 8444-254 (this conference).

[12] Canestrari, R., et al., "Techniques for the manufacturing of stiff and lightweight optical mirror panels based on slumping of glass sheets: concepts and results". Proc. SPIE 7437 (2009).

[13] Vandenbroucke J. for the CTA consortium, "Development of an ASIC for Dual Mirror Telescopes of the Cherenkov Telescope Array", $32^{\text {nd }}$ ICRC (2011). 